\title{
A unified approach to substructuring and structural modification problems
}

\author{
Walter D'Ambrogio ${ }^{\mathrm{a}, *}$ and Aldo Sestieri ${ }^{\mathrm{b}}$ \\ ${ }^{a}$ Università de L'Aquila, Dipartimento di Energetica, Località Monteluco, I-67040 Roio Poggio (AQ), Italy \\ ${ }^{\mathrm{b}}$ Universit di Roma "La Sapienza”, Dipartimento di Meccanica e Aeronautica, Via Eudossiana 18, I-00184 Roma, \\ Italy
}

This paper is dedicated to Bruno Piombo. Bruno was a collegue of us, a good scientist who spent most of his research work on vibration, modal analysis and signal processing applications, contributing with new and original ideas. But, primarily, Bruno was a friend, an affectionate friend of many of us involved in modal analysis and attending so many conferences together. His departure will deny us of his humor, profundity and respect, and left a gap which will be hard to fill. We think that this modest contribution is a way to remember Bruno to those who loved him but also to those who had not the chance of knowing him.

\begin{abstract}
Substructures coupling is still an important tool in several applications of modal analysis, especially structural modification and structures assembling. The subject is particularly relevant in virtual prototyping of complex systems and responds to actual industrial needs. This paper analyzes the possibility of assembling together different substructures' models. The important role of rotational DoFs is highlighted, underlying the difficulty of assembling theoretical and experimental models, for which, usually, the rotational DoFs are not available. Expansion techniques can be used to provide this information as well as appropriate modelling of joints. With this information FRF models, modal models and FE models can be appropriately combined together and solutions for several cases of practical interest are presented. The analyzed procedures are tested on purpose-built benchmarks, showing limits and capabilities of each of them.
\end{abstract}

\section{Introduction}

A major need in applied research that is increasingly accepted as a valid stimulus in the scientific community is the possibility of using different models of substructures in the process of assembling complex systems. This possibility, besides answering production needs, permits to perform local (substructure) optimizations and, in the final stage, an overall optimization. Moreover, the possibility of using different models, obtained from a theoretical or numerical analysis such as a Finite Element Model (FEM) or derived from experimental tests (Frequency Response Functions: FRFs) provides the chance of performing a virtual prototyping of the system, eventually permitting structural modifications of single components when the overall dynamic response of the assembled system is not satisfactory.

It is worthwhile to point out clearly that, when speaking about substructures' coupling, two different problems can be actually considered. The first one refers typically to substructures that must be assembled together through a number of joints. The second case involves a base structure that is coupled to a second system with the aim of performing a structural modification to change the dynamic behaviour of the base structure. Although there is not a substantial distinction between these types of coupling, in practice these two problems have been always considered

\footnotetext{
*Corresponding author. E-mail: dambro@ing.univaq.it.
} 
separately, for several reasons. In particular the problem of structural modification was traditionally dealt as a specific branch of modal analysis in that originally only the problem of lumped modifications was developed.

Dynamic models of structures can be classified according both to the type and to the source of information that defines each model. With regard to the source, it is possible to distinguish between theoretical models and experimental models, whilst relatively to the type it is possible to distinguish between physical models (characterized by elasticity, inertia and eventually damping properties), modal models (characterized by natural frequencies, vibration modes and modal dampings) and FRF models (described by an input-output relation in the frequency domain). Overall, six different combinations exist, that are summarized in the following Table, where the chance of occurrence of each combination is indicated.

\begin{tabular}{|c|c|c|c|}
\hline \multirow{4}{*}{ Origin $\left\{\begin{array}{l}\text { Theoretical } \\
\text { Experimental }\end{array}\right.$} & \multicolumn{3}{|c|}{$\underbrace{\text { Type }}$} \\
\hline & Physical & Modal & FRF \\
\hline & Always & Typical & Unusual \\
\hline & Never & Typical & Always \\
\hline
\end{tabular}

The coupling of structures described by different models - some by experimental FRFs, while others, especially those not yet constructed and potentially amenable to modification, by theoretical models - can be obtained using several procedures:

- coupling techniques using the FRF model, once the FRFs of the substructures have been experimentally determined;

- coupling techniques using the modal model, that can be applied once the substructure modes have been either identified from experimental FRFs or computed from an FE model;

- coupling techniques using the physical model, applicable when an FE model can be identified (e.g. at the end of a model updating process) from substructures whose FRF or modal model is known;

- coupling techniques using a hybrid approach, that uses the FRF or modal model of some structures and an FE model of others.

All the above methods are valid from a theoretical point of view, but any of them have peculiarities that make it more or less convenient for the particular case of interest or for a particular application.

The coupling using the FRF model has limitations related to the difficulty of measuring the rotational degrees of freedom (RDoFs), that are generally involved when joining together structures characterized by flexural behavior [13]. In fact, neglecting the RDoFs, an important part of continuity and equilibrium conditions at the joint, are excluded and the obtained results are generally meaningless. However, this approach is still appropriate when coupling together substructures whose dynamic behaviors are known experimentally by FRFs. Besides the limitation related to RDoFs, the coupling using the modal model [4,5] has additional drawbacks related to the identification of the modal parameters and to mode truncation. The hybrid coupling still presents the problem of the rotational degrees of freedom, while the coupling in the physical space does not have this limitation, but it implies that the physical model is known. In the last case, the process usually starts from an initial model of the substructures and ends with the model updating of some physical parameters to better reproduce the experimental dynamic behavior.

Furthermore, it should be recalled that substructures can be coupled together through several types of joints. Among them, two main categories can be distinguished:

- point connections, ideally involving one or several discrete points. These connections are typical of substructures coupled together through a finite set of joints as well as of lumped modifications. For such connections, the coupling conditions can be imposed at all the interface degrees of freedom, so that in principle an exact solution can be achieved;

- line and surface connections, involving a continuous interface along one or more ideal lines, or across one or more surfaces. These joints are typical of distributed structural modifications as well as of substructures joined along a line. For this types of connections, the coupling conditions can be imposed only at a discrete number of points, so that, in general, it is not possible to satisfy the coupling conditions along/across the continuous interface, unless this is described through appropriate shape functions. This issue is clearly mentioned in [7], where the coupling techniques that are unable to ensure a mathematically convergent solution in case of line/surface connections are referred to as heuristic. 
In all cases, the role of rotational degrees of freedom is crucial. At the state of the art, experimental testing is not able to provide FRFs containing rotational responses and moments. Although it is possible now to obtain rotational responses, albeit difficult to perform [6] and non standard [8-10], the problem of making tools capable of exciting the structure with concentrated moments is still far from a satisfactory solution [11-13]. Therefore it is necessary to provide an approximate description of the coupling mechanism trying to eliminate (condense) or expand the RDoFs.

However, once the importance of RDoFs is totally recognized, it is normal expectation to analyze whether the description of coupling conditions could be achieved without using such DoFs, at least under particular conditions. It is a matter of fact that several industrial problems are so complex that a satisfactory application of expansion techniques is hardly achieved either because the problem conditioning is very bad or because good measurements are difficult to obtain. Therefore an attempt is made to overcome the problem of estimating the RDoFs, by proposing a particular model of the joint: to this end the coupling condition is described by an equivalent model that does not transmit concentrated moments and rotations, i.e. does not need RDoFs. The results obtained by this analysis are quite satisfactory, at least in the range of low frequencies, when using both simulated and experimental data.

\section{Coupling techniques}

In the wide range of coupling techniques so far proposed in the literature, it is possible to extract four main important sets:

- FRF coupling techniques;

- modal coupling techniques;

- FE (physical) coupling techniques;

- hybrid coupling techniques, that can be further subdivided into:

* modal based hybrid coupling;

* FRF based hybrid coupling.

In the first group the mathematical model used for the structures is the FRF model; in the second group the mathematical model is the modal model for both substructures; in the third it is the physical (FE) model for both substructures; in the fourth the mathematical model is the physical model for one substructure, whilst for the other substructure the model can be either the modal or the FRF model.

The discussion in this paper will not be addressed to FE coupling techniques, physical models being essentially used within Finite Element software packages.

\subsection{FRF coupling techniques}

It is first necessary to divide the total set of DoFs of each substructure into internal DoFs ( $i$ ), not belonging to the joints, and coupling DoFs $(c)$, that are those participating to the joint region.

It is assumed that the two substructures (A and B) are known through their FRF matrices, which can be partitioned into internal DoFs $i$ and coupling DoFs $c$ :

$$
\mathbf{H}^{A}(\omega)=\left[\begin{array}{c}
\mathbf{H}_{i i}^{A} \mathbf{H}_{i c}^{A} \\
\mathbf{H}_{c i}^{A} \mathbf{H}_{c c}^{A}
\end{array}\right] \quad \mathbf{H}^{B}(\omega)=\left[\begin{array}{c}
\mathbf{H}_{i i}^{B} \mathbf{H}_{i c}^{B} \\
\mathbf{H}_{c i}^{B} \mathbf{H}_{c c}^{B}
\end{array}\right]
$$

Let $\mathbf{u}^{A}, \mathbf{u}^{B}$ be the displacements and rotations of substructures $\mathrm{A}$ and $\mathrm{B}$, respectively. Furthermore, let $\mathbf{f}^{A}, \mathbf{f}^{B}$ and $\mathbf{f}$ be the forces and moments acting on subsubstructures $A$ and $B$, and on the coupled structure, respectively. The compatibility of displacements and rotations, and the equilibrium of forces and moments at the coupling DoFs $c$ can be expressed as:

$$
\mathbf{u}_{c}^{A}=\mathbf{u}_{c}^{B} ; \quad \mathbf{f}_{c}^{A}+\mathbf{f}_{c}^{B}=\mathbf{f}_{c}
$$

This leads to the classical expression for the FRF of the coupled structure C: 


$$
\mathbf{H}^{C}=\left[\begin{array}{ccc}
{\left[\mathbf{H}^{A}\right]_{i i}^{-1}} & {\left[\mathbf{H}^{A}\right]_{i c}^{-1}} & 0 \\
{\left[\mathbf{H}^{A}\right]_{c i}^{-1}} & {\left[\mathbf{H}^{A}\right]_{c c}^{-1}+\left[\mathbf{H}^{B}\right]_{c c}^{-1}} & {\left[\mathbf{H}^{B}\right]_{c i}^{-1}} \\
0 & {\left[\mathbf{H}^{B}\right]_{i c}^{-1}} & {\left[\mathbf{H}^{B}\right]_{i i}^{-1}}
\end{array}\right]^{-1}
$$

In it three matrix inversions are involved (two inversions for the complete FRF matrices of each substructure plus the inversion of the assembled impedance matrix). Due to the number of matrix inversions and to the size of the involved matrices, ill-conditioning may easily occur, leading to large numerical errors.

\subsubsection{FRF coupling: the algorithm by Jetmudsen, Bielewa and Flannely}

To reduce the number of matrix inversions and to improve possibly the problem conditioning, a different algorithm was developed by Jetmundsen et al. [14] and subsequently generalized [15], leading to the following expression for the FRF of the coupled structure:

$$
\mathbf{H}^{C}=\left[\begin{array}{ccc}
\mathbf{H}_{i i}^{A} & \mathbf{H}_{i c}^{A} & 0 \\
\mathbf{H}_{c i}^{A} & \mathbf{H}_{c c}^{A} & \mathbf{H}_{c i}^{B} \\
0 & \mathbf{H}_{i c}^{B} & \mathbf{H}_{i i}^{B}
\end{array}\right]-\left\{\begin{array}{c}
\mathbf{H}_{i c}^{A} \\
\mathbf{H}_{c c}^{A} \\
\mathbf{H}_{i c}^{B}
\end{array}\right\} \times\left[\mathbf{H}_{c c}^{A}+\mathbf{H}_{c c}^{B}\right]^{-1}\left\{\begin{array}{l}
\mathbf{H}_{i c}^{A} \\
\mathbf{H}_{c c}^{A} \\
\mathbf{H}_{i c}^{B}
\end{array}\right\}^{T}
$$

which involves one matrix inversion only. Furthermore, the size of the matrix to invert is given by the number of interface DoFs. (In the classical procedure, the size of the FRF matrices to invert is given by the number of DoFs of each substructure and the size of the final inversion is given by the number of DoFs of the assembled structure.) Although a smaller size and a lower number of inversions does not ensure necessarily that the final conditioning of the problem improves, this is generally very probable.

To avoid inconsistencies of the FRF model and to reduce the number of measurements necessary to define an FRF matrix, it is beneficial to measure just a row or column of the FRF instead of the entire matrix (or its lower or upper triangular part). Then, the modal parameters can be identified and the necessary FRFs can be synthesized in the required frequency range. To reduce modal truncation effects, out of range modes can be accounted by using theoretically derived lower and upper residuals, for instance obtained from a raw FE model of each substructure.

\subsection{Modal coupling techniques: fixed versus free interface methods}

In modal coupling techniques, a reduced number of principal coordinates is used to describe each substructure through a Ritz-type transformation. Generally, higher order vibration modes are discarded. According to the choice of such principal coordinates, it is possible to distinguish two groups of methods: fixed interface methods and free interface methods.

In fixed interface methods [5], the total displacement of each substructure is obtained as a superposition of fixed interface normal modes plus constraint modes, i.e. static displacements caused by a unit displacement of each connection DoF, with other interface DoFs fixed. Fixed interface methods are easy to implement whenever mass and stiffness matrices of the substructures are known theoretically, i.e. by an FE model. However, they are difficult to apply when dealing with experimental data: in fact it is almost impossible to build up a perfectly fixed support for dynamic tests; furthermore, the influence coefficients from which constraint modes could be derived are also hardly obtained by static or dynamic testing.

In free interface methods, such as [16-18], the total displacement of each substructure is obtained as a superposition of normal modes with free interface plus (according to the particular method) rigid body modes, attachment modes, inertia relief modes, etc. Free interface methods are the most appropriate when dealing with experimental data, since the necessary vibration modes are obtained from a substructure with free interface or even freely supported, the condition most commonly used in modal testing. 


\subsection{Hybrid coupling: modal based versus FRF based techniques}

It is assumed that substructure $\mathrm{A}$ is known either through the experimental FRF matrix $\mathbf{H}^{A}(\omega)$ or through identified modal parameters $\Lambda^{A}$ and $\Phi^{A}$. On the contrary, substructure $\mathrm{B}$ is known through an FE model providing mass and stiffness matrices $\Delta \mathbf{M}^{B}$ and $\Delta \mathbf{K}^{B}$. It can be pointed out that $\Delta \mathbf{M}^{B}$ and $\Delta \mathbf{K}^{B}$ must represent the difference between the mass and the stiffness matrices of the coupled structure $(A+B)$ and those of substructure $A$, hence the use of the symbol $\Delta$. In fact, in many cases, it is not sufficient to consider the mass and stiffness matrices of substructure $\mathrm{B}$ alone. For instance, it is easy to realize that, when coupling a beam A with a reinforcing rib B to increase the thickness of the structure, the bending stiffness of the coupled (thicker) structure is not just the sum of the bending stiffness of the two components, but it is a more complex combination of both. Thus, the need of considering $\Delta \mathbf{M}^{B}$ and $\Delta \mathbf{K}^{B}$.

For the sake of simplicity, it is further assumed that substructure B has no internal DoFs, i.e. all the DoFs of substructure B are coupling DoFs $c: \Delta \mathbf{M}^{B}=\Delta \mathbf{M}_{c c}^{B}, \Delta \mathbf{K}^{B}=\Delta \mathbf{K}_{c c}^{B}$.

\subsubsection{Hybrid coupling using the modal database}

After enforcing the compatibility of displacements (rotations) and the equilibrium of forces (moments), the equation of free undamped motion of the coupled structure (N DoFs), partitioned into internal DoFs $i$ and coupling DoFs $c$, is:

$$
\left[\begin{array}{cc}
\mathbf{M}_{i i}^{A} & \mathbf{M}_{i c}^{A} \\
\mathbf{M}_{c i}^{A} & \mathbf{M}_{c c}^{A}+\Delta \mathbf{M}^{B}
\end{array}\right]\left(\begin{array}{c}
\ddot{\mathbf{u}}_{i}^{A} \\
\ddot{\mathbf{u}}_{c}
\end{array}\right)+\left[\begin{array}{cc}
\mathbf{K}_{i i}^{A} & \mathbf{K}_{i c}^{A} \\
\mathbf{K}_{c i}^{A} & \mathbf{K}_{c c}^{A}+\Delta \mathbf{K}^{B}
\end{array}\right]\left(\begin{array}{c}
\mathbf{u}_{i}^{A} \\
\mathbf{u}_{c}
\end{array}\right)=0
$$

The mass and stiffness matrices of substructure $\mathrm{A}, \mathbf{M}^{A}$ and $\mathbf{K}^{A}$, are not available. By ideally solving the eigenvalue problem $\left(\mathbf{K}^{A}-\lambda \mathbf{M}^{A}\right) \phi=0, N$ eigenvalues $\Lambda^{A}=\operatorname{diag}\left(\omega_{n_{1}}^{2}, \ldots, \omega_{n_{N}^{2}}\right)$ and eigenvectors $\Phi^{A}=$ $\left[\begin{array}{llll}\phi_{1}^{A} & \phi_{2}^{A} & \ldots & \phi_{N}^{A}\end{array}\right]$ are obtained, that satisfy the orthonormal conditions $\Phi^{A^{T}} \mathbf{M}^{A} \Phi^{A}=\mathbf{I}$ and $\Phi^{A^{T}} \mathbf{K}^{A} \Phi^{A}=\Lambda^{A}$. By introducing the coordinate transformation $\Phi^{A}$ from the physical displacements $\mathbf{u}^{A}$ to the modal coordinates $\mathbf{q}$ of substructure A, one obtains, after pre-multiplying by $\Phi^{A^{T}}$ and in view of the orthonormal conditions:

$$
\left(\mathbf{I}+\Phi_{c}^{A^{T}} \Delta \mathbf{M}^{B} \Phi_{c}^{A}\right) \ddot{\mathbf{q}}+\left(\Lambda^{A}+\Phi_{c}^{A^{T}} \Delta \mathbf{K}^{B} \Phi_{c}^{A}\right) \mathbf{q}=0
$$

where $\Phi_{c}^{A}$ is the mode shape matrix at the coupling DoFs $c$.

Note that $\Delta \tilde{\mathbf{M}}=\Phi_{c}^{A^{T}} \Delta \mathbf{M}^{B} \Phi_{c}^{A}$ and $\Delta \tilde{\mathbf{K}}=\Phi_{c}^{A^{T}} \Delta \mathbf{K}^{B} \Phi_{c}^{A}$ are not usually diagonalized by the coordinate transformation $\Phi^{A}$ based on the eigenvectors of substructure A only. To obtain eigenvalues and eigenvectors of the coupled structure, the eigenvalue problem associated with Eq. (6) must be solved. Eq. (6) does not contain the mass and stiffness matrices of substructure $\mathrm{A}$, but requires the modal parameters $\Lambda^{A}$ and $\Phi^{A}$, which are assumed to be known. For practical reasons, a number $m \ll N$ of modes is used, so that the matrices in Eq. (6) are truncated to $m$ rows and $m$ columns, and the modal parameters of the modified structure are not determined exactly, but are approximate values (modal truncation problem). Furthermore, the mode shapes might be known only on a subset of the coupling DoFs (spatial incompleteness), calling for reduction or expansion techniques. In fact, Eq. (6) requires $\Phi_{c}^{A}, \Delta \mathbf{K}^{B}$ and $\Delta \mathbf{M}^{B}$ to be referred to the same set of DoFs.

\subsubsection{Hybrid coupling using the FRF database}

The relationship providing the FRF of the coupled structure is given in [19]:

$$
\mathbf{H}^{C}(\omega)=\left[\begin{array}{c}
\mathbf{H}_{i i}^{A} \mathbf{H}_{i c}^{A} \\
\mathbf{H}_{c i}^{A} \mathbf{H}_{c c}^{A}
\end{array}\right]-\left[\begin{array}{c}
\mathbf{H}_{i c}^{A} \\
\mathbf{H}_{c c}^{A}
\end{array}\right] \times \Delta \mathbf{Z}_{c c}^{B}\left[\mathbf{I}_{c c}+\mathbf{H}_{c c}^{A} \Delta \mathbf{Z}_{c c}^{B}\right]^{-1}\left[\begin{array}{ll}
\mathbf{H}_{c i}^{A} & \mathbf{H}_{c c}^{A}
\end{array}\right]
$$

where $\mathbf{H}^{C}$ is the FRF of the coupled structure, and $\Delta \mathbf{Z}^{B}=\Delta \mathbf{K}^{B}+j \omega \Delta \mathbf{C}^{B}-\omega^{2} \Delta \mathbf{M}^{B}$ is the dynamic stiffness matrix due to the addition of substructure $B$.

In view of Eq. (7), the matrices $\mathbf{H}_{c c}^{A}$ and $\Delta \mathbf{Z}^{B}$ must be referred to the same set of DoFs. 


\section{Techniques to account for rotational DoFs}

Whatever the coupling technique (impedance, modal or hybrid), experimental testing does not usually involve rotational DoFs. Hence the need of expanding experimentally determined mode shapes to include unmeasured rotational DoFs (SEREP expansion or static expansion). Just for hybrid coupling, an alternative is to perform a sort of dynamic reduction, involving the local models of the two substructures [20].

A further alternative can be to avoid the use of rotational DoFs by assuming that a rigid connection at a given point - involving displacement and rotation compatibility, and forces and moments equilibrium - can be substituted by an equivalent connection through several close points - involving only displacement compatibility and force equilibrium (translational DoFs only).

\subsection{SEREP expansion}

In SEREP (System Equivalent Reduction Expansion Technique [21]) the expanded mode shapes (including the rotational DoFs) are obtained from the experimental modes using the transformation matrix:

$$
\mathbf{T}=\left[\begin{array}{l}
\Phi_{A 1} \\
\Phi_{A 2}
\end{array}\right]\left(\Phi_{A 1}^{T} \Phi_{A 1}\right)^{-1} \Phi_{A 1}^{T} \Longrightarrow\left[\begin{array}{l}
\Phi_{E 1} \\
\Phi_{E 2}
\end{array}\right]=\mathbf{T} \Phi_{X 1}
$$

where the subscripts A, X and E denote analytical, experimental and expanded modes, respectively, and the subscripts 1 and 2 refer to measured (some translational) DoFs and unmeasured (rotational plus the remaining translational) DoFs, respectively.

The main advantage of SEREP when used as an expansion technique is that the expanded modes are a linear combination of the analytical modes, so that measured data are in some sense smoothed out by the procedure. The main disadvantage of SEREP is that an FE model is required to obtain the analytical modes, and the quality of such model is crucial for the success of the procedure.

Moreover, the SEREP technique has some well known advantages when used for model reduction: invariance of the selected mode shapes and natural frequencies when passing from the full to the reduced model, and insensitivity of the quality of the results to the set of selected modes and DoFs.

\subsection{Static expansion using a local interface model}

In the static expansion using a local interface model [22,23], a local model of substructure A, i.e. a model including the interface region common to both the structures, can be considered ( $L$ DoFs, $c \subseteq L$ ). Then, $\mathbf{u}_{c}=\mathbf{T}_{c L} \mathbf{u}_{L}$, with $\mathbf{T}_{c L}$ boolean, and equal to $\mathbf{I}$ if $c \equiv L$. DoFs $L$ can be partitioned into test DoFs (subscript 1 ) and unmeasured DoFs (subscript 2), so that the stiffness and mass matrices are:

$$
\mathbf{K}_{L L}^{A}=\left[\begin{array}{ll}
\mathbf{K}_{11}^{A} & \mathbf{K}_{12}^{A} \\
\mathbf{K}_{21}^{A} & \mathbf{K}_{22}^{A}
\end{array}\right] \quad \mathbf{M}_{L L}^{A}=\left[\begin{array}{ll}
\mathbf{M}_{11}^{A} & \mathbf{M}_{12}^{A} \\
\mathbf{M}_{21}^{A} & \mathbf{M}_{22}^{A}
\end{array}\right]
$$

By using the static (Guyan) condensation over the test DOFs 1, one obtains:

$$
\mathbf{u}_{L}=\left[\begin{array}{c}
\mathbf{I} \\
-\mathbf{K}_{22}^{A^{-1}} \mathbf{K}_{21}^{A}
\end{array}\right] \mathbf{u}_{1}=\mathbf{T}_{L 1} \mathbf{u}_{1}
$$

The Guyan transformation is exact under the assumpion that inertia actions are zero and that no actions are applied at DoFs 2. In practice, results are acceptable provided that negligible actions are exchanged at the unmeasured DoFs 2 and that inertia actions are negligible too.

Therefore the expanded mode shapes at the coupling DoFs $\Phi_{c}^{A}$ can be obtained from experimental modes $\Phi_{X 1}^{A}$ using the transformation matrix:

$$
\mathbf{T}_{c 1}=\mathbf{T}_{c L} \mathbf{T}_{L 1}=\mathbf{T}_{c L}\left[\begin{array}{c}
\mathbf{I} \\
-\mathbf{K}_{22}^{A^{-1}} \mathbf{K}_{21}^{A}
\end{array}\right] \Longrightarrow \Phi_{E c}^{A}=\mathbf{T}_{c 1} \Phi_{X 1}^{A}
$$

which could be used immediately for expansion. A different and more effective transformation matrix can be obtained if a further step is taken in order to smooth experimental mode shapes so as to reduce the effect of bad 
sensors. Smoothing can be performed by expressing the displacement at the test DoFs through a truncated Ritz basis made by $N_{G}<N_{1}$ eigenvectors $\tilde{\Phi}$ (interface modes) of the local model of substructure A, statically condensed to the test DoFs, i.e.:

$$
\mathbf{u}_{1_{s}}=\tilde{\Phi} \mathbf{q}_{G}
$$

where the subscript $s$ stands for smoothed and $\mathbf{q}_{G}$ is found so as to minimize $\left\|\mathbf{u}_{1}-\mathbf{u}_{1 s}\right\|$. The final form of the transformation matrix is [23]:

$$
\mathbf{T}_{c 1_{s}}=\mathbf{T}_{c L} \mathbf{T}_{L 1} \tilde{\Phi}\left(\tilde{\Phi}^{T} \tilde{\Phi}\right)^{-1} \tilde{\Phi}^{T}
$$

An indicator of the accuracy of the procedure is also presented in [23]. It is necessary to compute the first natural frequency of substructure A for fixed test DoFs: such frequency must be high enough when compared with the frequency range of interest.

The main advantage of the expansion using a local interface model is that, unlike in SEREP, a fairly accurate FE model of the entire substructure is not required, but only a model of the region involved with the joint is necessary. This kind of expansion can be efficiently used whenever the joint between the substructures extends across a surface, as in the distributed structural modification case.

\subsection{Dynamic reduction using a quasi-local model}

A dynamically reduced dynamic stiffness matrix $\Delta \tilde{\mathbf{Z}}^{B}$, including the contribution of substructure B can be obtained as:

$$
\Delta \tilde{\mathbf{Z}}^{B}=\tilde{\mathbf{Z}}-\tilde{\mathbf{Z}}^{A}
$$

where $\tilde{\mathbf{Z}}$ and $\tilde{\mathbf{Z}}^{A}$ are the dynamically reduced dynamic stiffness matrices of the coupled structure and of substructure A, respectively. In [20] it is shown that, because of the quasi-local character of $\Delta \tilde{\mathbf{Z}}$, the difference can be approximated by using a local model of substructure A, that must include obviously the coupling DoFs $c$. The suggested approach is:

- a local model of substructure A is considered, providing $\mathbf{M}_{L L}^{A}, \mathbf{K}_{L L}^{A}\left(\right.$ and $\left.\mathbf{C}_{L L}^{A}\right)$;

- a local model of the coupled structure is also considered, providing $\mathbf{M}_{L L}, \mathbf{K}_{L L}$ (and $\mathbf{C}_{L L}$ ). These matrices, as those of the previous item, include unmeasured DoFs;

- dynamic reduction is performed on the two previous models, yielding the reduced dynamic stiffness matrices $\tilde{\mathbf{Z}}$ and $\tilde{\mathbf{Z}}^{A}$. This operation introduces some approximation;

- the difference between the two previous matrices provides the reduced dynamic stiffness matrix, that can be used to determine the FRF of the coupled structure through Eq. (7).

\subsection{Interface model without RDoFs: equivalent multi-point connection}

When a rigid connection between two structures is considered at a given point, the coupling conditions involve equilibrium of both forces and moments, and compatibility of both displacements and rotations. If coupling is assumed through a single point, neglecting rotational DoFs provides meaningless results. However, if coupling is rather assumed through several close points, some information about rotations and moments can be implicitly recovered from forces and displacements, even if rotational DoFs are neglected.

For a joint between two parallel plates subjected to bending, coupling can be assumed through three points of the joint region, not lying on the same straight line - e.g. at a triangle's vertices. Only translational DoFs orthogonal to the joint are considered. This amounts to assume that, at these points, only forces orthogonal to the plates are transmitted. The combination of such forces provides a resultant force and a resultant moment approximating the real dynamic actions exchanged between the coupled structures. In terms of joint's displacement and rotation compatibility, the use of translational DoFs only at the three points requires that - for a reasonable accuracy of the procedure - the joint region lies approximately on a plane. In this case, from the values of the three displacements, the average displacement and the rotation of the joint plane could be implicitly obtained.

The limitation of this approach is related to the ratio between the points distance in the joint and the frequency (wavelength) of interest: when a quarter of the involved wavelength equals the points distance, the joint region is not anymore approximated by a plane and the procedure becomes more and more inaccurate. 


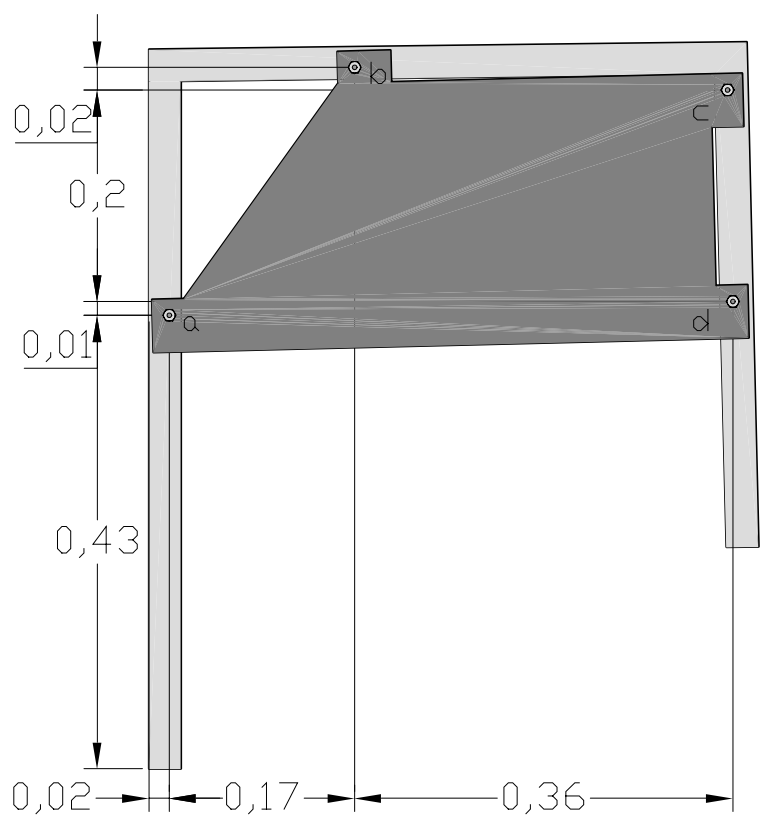

Fig. 1. Frame and plate coupled along four regions.

\subsection{Discussion about expansion techniques}

A number of advantages and disadvantages of the presented expansion techniques have been already summarized in the description of each technique. However, further comments can be made, particularly about which type of joint is more suitably described by each technique.

SEREP expansion is well suited both for point connections and for line and surface connections, but, as mentioned previously, it has the disadvantage of requiring a fairly accurate FE model of the entire substructure, which is often in contrast with the premise of this work that assumes that some substructures are difficult to model theoretically.

Methods based on local models (static expansion and dynamic reduction) are well suited for line and surface connections: in this case the local model is able to capture the essential characteristics of the interface behavior.

Finally, the technique based on the equivalent multi-point connection is well suited for point connections, as long as the interface around each connection can be reasonably thought to lie on a plane.

\section{Results and comparison on benchmarks}

Two benchmark structures are considered to test the reliability of the different coupling techniques on both types of joints. The first benchmark is characterized by a coupling that ideally involves four discrete point connections, whilst the second benchmark involves coupling across a surface (continuously distributed structural modification).

\subsection{FRF coupling of a plate-frame benchmark}

The benchmark is made of a plate and a frame, both in aluminium. The two substructures are supposed to be coupled along four regions as shown in Fig. 1 that includes the dimensions of the coupled system.

At a first stage, both substructures were modelled by finite elements to check, step by step, the different phases of the coupling techniques, acquiring, in such a way, a particular sensitivity on each problem. First the analysis was performed without introducing any kind of error in the data and then polluting them with Gaussian random noise, enlightening a set of different drawbacks and showing when the procedures are able to produce good results or not. 
Table 1

Experimental vs. theoretical natural frequencies of the frame using beam elements

\begin{tabular}{lccccc}
\hline Natural frequencies of the frame [Hz] & Mode 1 & Mode 2 & Mode 3 & Mode 4 & Mode 5 \\
\hline Experimental & 14.79 & 45.67 & 68.15 & 108.19 & 110.81 \\
FE beam model of the frame & 19.10 & 45.69 & 71.70 & 115.51 & 120.41 \\
\hline
\end{tabular}

Table 2

Experimental vs. theoretical natural frequencies of the frame using shell elements

\begin{tabular}{lccccc}
\hline Natural frequencies of the frame [Hz] & Mode 1 & Mode 2 & Mode 3 & Mode 4 & Mode 5 \\
\hline Experimental & 14.79 & 45.67 & 68.15 & 108.19 & 110.81 \\
FE shell model of the frame & 14.95 & 47.15 & 70.17 & 111.61 & 118.51 \\
\hline
\end{tabular}

At a second stage, experimental tests were performed and the coupling techniques were applied to these data to check the problems arising when working with real data: issues concerning ill-conditioning, level of noise, how many and which degrees of freedom to measure were analysed.

By the first analysis it was observed that a correct description of the frame (as verified by comparing the model with experimental tests) cannot be obtained by beam elements because such FE model produces some natural frequencies that are in error by about $50 \%$ with respect to the experimental data (see Table 1). In fact, the cross-sections of the beam elements remain plane and parallel during the torsion. In other words, with such elements, the torsion of the intermediate horizontal beam induces displacements of the two vertical beams along the normal direction to the structure plane ( $z$ direction) only, whilst displacements along the $x$ direction (parallel to the intermediate beam) are also experimentally observed. Such displacements are allowed by the shell elements. Therefore it was necessary to use shell elements for both the frame and the plate, obtaining much better results as seen in Table 2.

The FE model of the plate, obtained by shell elements, did not show any problem and the results of the model fitted quite precisely the experimental data.

Once obtained a correct FE model of both substructures, a detailed analysis of the FRF coupling technique was developed. When using simulated data, it was assumed that only the translational FRFs of both structures were known, as those practically available from experiments.

\subsubsection{FRF coupling with expansion of translational DoFs using SEREP}

A first analysis is addressed to the FRF coupling procedure that, for a correct application, needs that the rotational FRFs (rotation/force, translation/moment, rotation/moment) are estimated. This estimate is obtained by an expansion technique using SEREP, that requires the theoretical mode shapes (FE) of the component substructures. The FE model, that must be purpose-built for the application of SEREP, should satisfy two conflicting requirements: on one side it should not be too complex, while, on the contrary, it should provide accurate modes to avoid significant errors in the expansion.

With this in mind, the sensitivity to a decrease of the model accuracy - i.e. to a decrease of the number of DoFs in the FE mesh - is evaluated. When using simulated data, it is observed that a small decrease of the model accuracy of both substructures induces relevant errors, making such procedure very sensitive to the quality of the model.

However, when applying the same technique to FRFs determined experimentally for both the plate and the frame, which is expected to be the most critical case, the results of the coupling are incredibly in good agreement with reference FRFs (Figs 2 and 3).

The conditioning of the modal matrices that must be inverted for the application of SEREP is also analyzed. The FRFs of the plate and the frame are measured at seven points - the only measured degree of freedom being the acceleration along the transverse direction $z$. In the measured frequency range, seven modes are identified for the plate and six modes for the frame. Since the SEREP expansion needs a determined or overdetermined matrix (Eq. (5)), the minimum number of test points is seven. The use of more test points worsens the conditioning of the matrix to invert, and probably leads to a SEREP transformation matrix much more sensitive to errors in the identified mode shapes. Figs 4 and 5 show a reconstructed acceleration/moment FRF element of the frame, using 7 test points and 10 test points, respectively. Results are compared with the theoretical FRF. It can be noticed that the FRF expanded using 7 points is more accurate than the other one. 


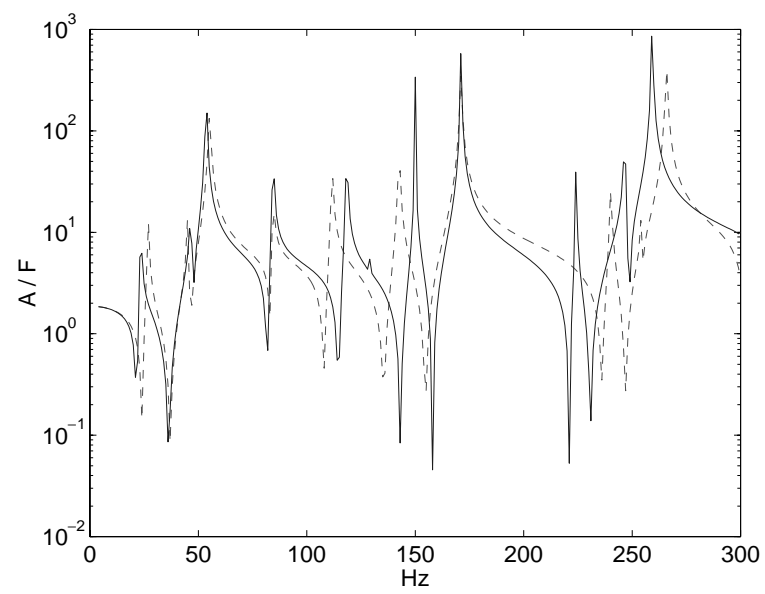

Fig. 2. Frame-plate coupling. Translation/force FRFs: complete FE model (-); coupling procedure (- - -) using experimental data.

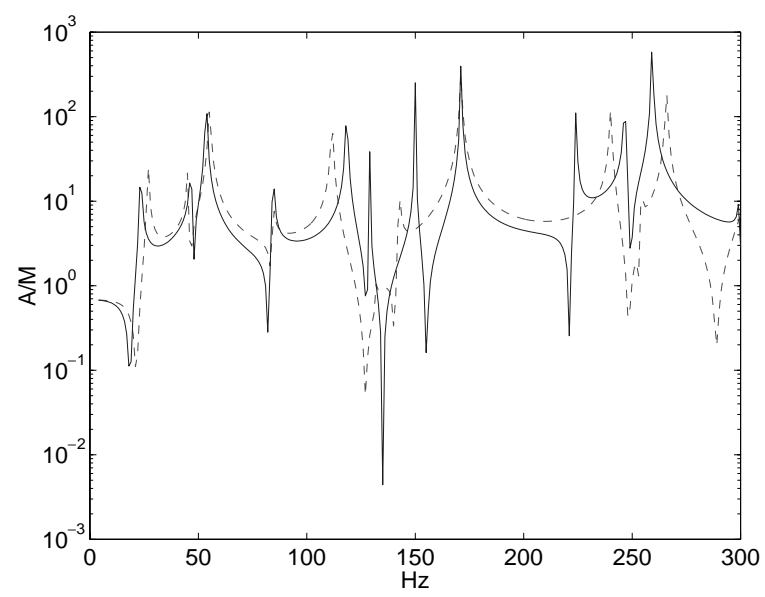

Fig. 3. Frame-plate coupling. Translation/moment FRFs: complete FE model (-); coupling procedure (- - -) using experimental data.

\subsubsection{FRF coupling: equivalent multi-point connection}

Whether or not expansion techniques to estimate the RDoFs necessary to perform the FRF coupling can be effectively used, one is tempted to analyze whether an appropriate description of the interface region can be obtained without using the rotational DoFs, as outlined previously. This approach would avoid the expansion operation that in any case depends on several factors difficult to control.

To this aim, three non aligned points in the interface region were considered for coupling. The maximum distance between such points was about $21 \mathrm{~mm}$. The only DoFs retained for coupling were those orthogonal to the joint.

The results obtained from this type of analysis were quite satisfactory, when using both noise polluted simulated data (not reported in the paper) and experimental data, as can be observed from Fig. 6 where the reference FRF of the coupled system is compared with the coupled FRF obtained by using the measured translational DoFs only. The agreement is very good, at least for low frequencies, that, in any case, are the most interesting in structural dynamics. The result confirms that the limitation of this approach is related to the ratio between the points distance in the joint and the frequency (wavelength) of interest: for the given points distance the upper limiting frequency can be estimated at about $150 \mathrm{~Hz}$.

Furthermore, it can be stressed that this procedure is much less sensitive to random noise and systematic modelling errors, and, of course, it is computationally much more convenient in that it does not need the application of expansion procedures such as SEREP. 


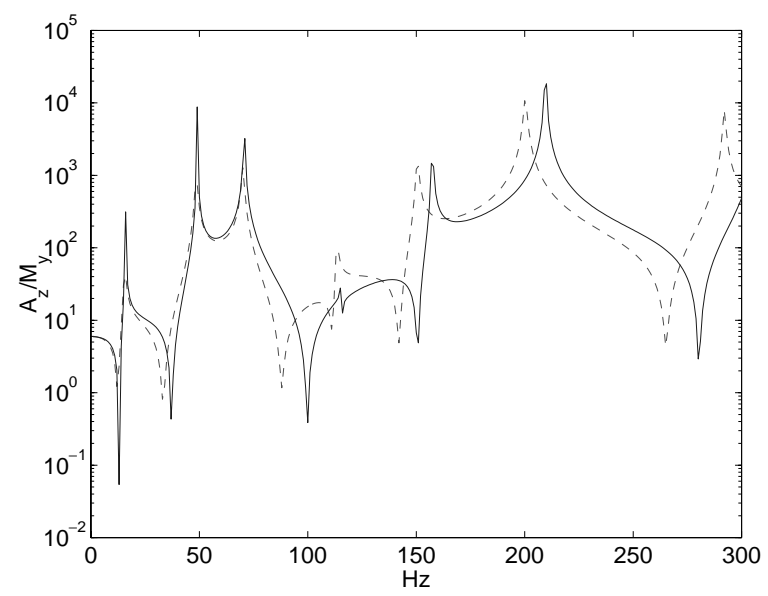

Fig. 4. SEREP expansion of translation/moment frame FRFs: theoretical FRF from FE model (-); SEREP expansion using 7 test points (- - -).

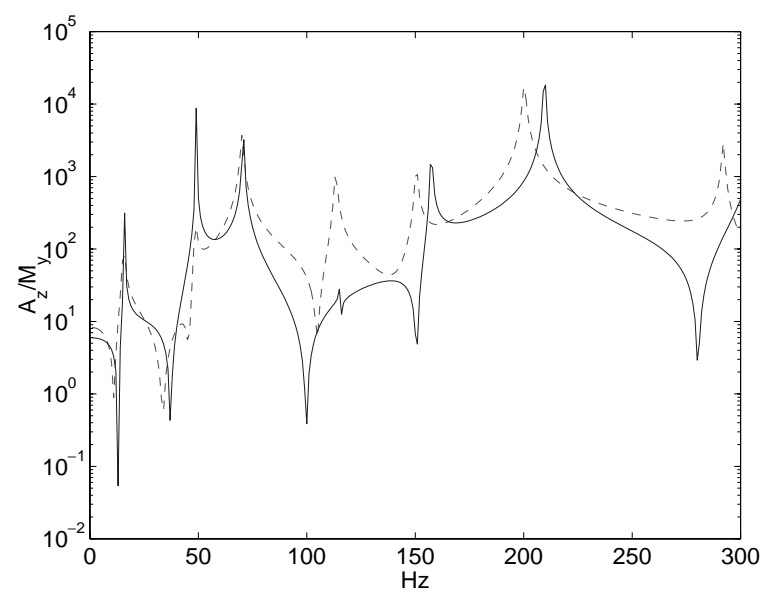

Fig. 5. SEREP expansion of translation/moment frame FRFs: theoretical FRF from FE model (-); SEREP expansion using 10 test points (- - -).

\subsection{Hybrid coupling of a plate-reinforcing plate benchmark}

The benchmark consists in one aluminium plate, with size $600 \mathrm{~mm} \times 800 \mathrm{~mm} \times 3 \mathrm{~mm}$. Another aluminium plate, with the same thickness, is coupled to the first plate across its surface. The second plate is smaller than the first one and is bolted to it. In Fig. 7(a) draft of the structure under test is presented.

As a first step for simulation, a finite element model of the larger plate is built to perform preliminary computations assuming free-free support conditions. The model is built with four node shell elements with 6 DoFs per node by means of the ANSYS code. The mesh of the model is $50 \mathrm{~mm} \times 50 \mathrm{~mm}$ for a total number of 1326 DoFs.

The frequency range of interest is $0-150 \mathrm{~Hz}$ in which 10 flexible modes are found. In Table 3 the natural frequencies computed by the finite element model are compared to those obtained by experimental tests on the base structure.

Another finite element model is built for the coupled structure and the same elements and mesh dimensions are used as in the original structure for the same total number of DoFs. This model is used as a reference to compare results obtained using simulated data.

To obtain $\Delta \mathbf{K}^{B}$ and $\Delta \mathbf{M}^{B}$, two more local FE models are built to describe the interface region of the base plate and of the coupled structure, using four node plate elements with 3 DoFs per node. In this case a coarser mesh, $100 \mathrm{~mm} \times 100 \mathrm{~mm}$, is used, for a total number of 36 DoFs. The related code is written in Matlab and it is used within the algorithm which implements the whole procedure. 
Table 3

Finite Element vs. experimental natural frequencies of the base plate

\begin{tabular}{|c|c|c|}
\hline \multicolumn{3}{|c|}{ Natural Frequencies of the base structure $[\mathrm{Hz}]$} \\
\hline & F.E. model & Experimental \\
\hline Mode 1 & 20.60 & 19.6 \\
\hline Mode 2 & 24.52 & 24.8 \\
\hline Mode 3 & 45.56 & 45.5 \\
\hline Mode 4 & 48.85 & 47.2 \\
\hline Mode 5 & 59.64 & 58.0 \\
\hline Mode 6 & 71.64 & 72.4 \\
\hline Mode 7 & 93.43 & 91.3 \\
\hline Mode 8 & 99.64 & 96.4 \\
\hline Mode 9 & 124.52 & 124.5 \\
\hline Mode 10 & 137.86 & 138.8 \\
\hline
\end{tabular}

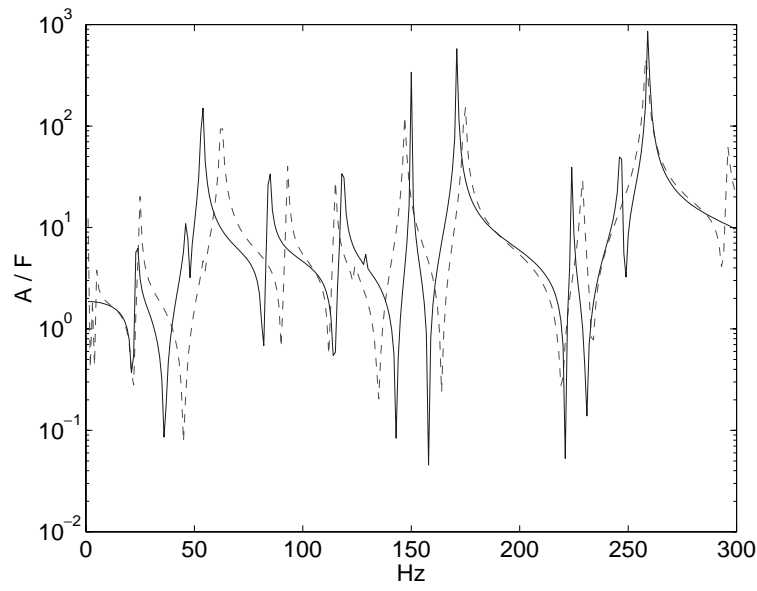

Fig. 6. Frame-plate coupling. Translation/force FRFs: complete FE model (-); coupling by omitting the rotational DoFs (- - -) and using test data

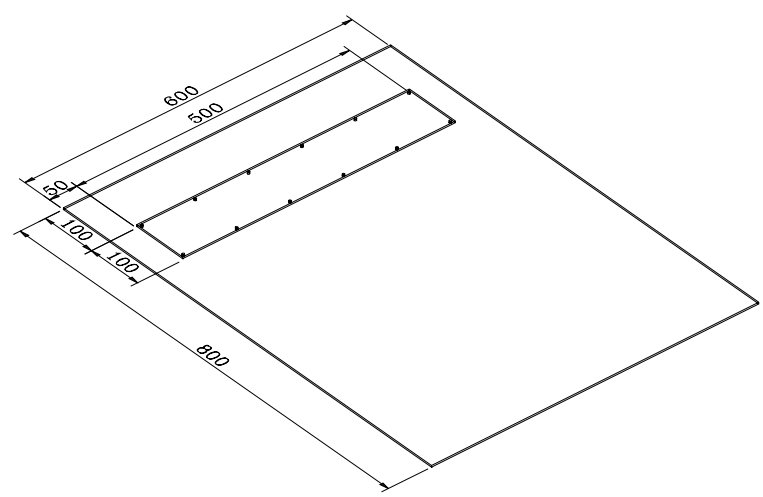

Fig. 7. Coupled structure: plate modified by a reinforcing plate.

Experimental tests are carried out by measuring the translational FRFs at the 12 points corresponding to the nodes of the region described by the local FE models.

\subsubsection{Hybrid coupling: local static expansion vs. dynamic reduction and SEREP}

The method is first applied using simulated data, then it is applied using data identified from experimental tests. Simulated data are obtained by the theoretical FE model of the base plate, which provides theoretical natural 
Table 4

Finite element vs. predicted natural frequencies of the coupled structure

\begin{tabular}{lrcccc}
\hline \multicolumn{5}{c}{$\begin{array}{c}\text { Natural frequencies of the base structure }[\mathrm{Hz}] \\
\text { Predicted }\end{array}$} \\
\cline { 3 - 6 } & F.E.M. & \multicolumn{4}{c}{} \\
\cline { 3 - 6 } Mode 1 & 22.25 & 22.07 & 22.35 & 22.65 & 21.19 \\
Mode 2 & 25.06 & 24.65 & 24.66 & 24.88 & 26.75 \\
Mode 3 & 51.41 & 51.34 & 51.88 & 52.56 & 51.82 \\
Mode 4 & 58.68 & 58.94 & 59.14 & 60.09 & 58.36 \\
Mode 5 & 72.08 & 68.35 & 68.19 & 68.68 & 69.49 \\
Mode 6 & 79.06 & 78.12 & 77.80 & 78.57 & 79.58 \\
Mode 7 & 109.00 & 109.80 & 110.44 & 107.82 & 111.59 \\
Mode 8 & 113.73 & 114.70 & 113.68 & 116.30 & 118.12 \\
Mode 9 & 131.64 & 130.97 & 130.99 & 131.50 & 130.58 \\
Mode 10 & 150.94 & 133.74 & 133.82 & 135.38 & 134.83 \\
\hline
\end{tabular}

Table 5

Identified vs. predicted natural frequencies of the coupled structure

\begin{tabular}{lcccc}
\hline & $\begin{array}{c}\text { Natural frequencies of the base structure [Hz] } \\
\text { Experimental }\end{array}$ & \multicolumn{3}{c}{ Predicted } \\
\cline { 3 - 5 } & & $\begin{array}{c}\text { Modal based } \\
\text { static exp. }\end{array}$ & $\begin{array}{c}\text { FRF based } \\
\text { dyn. red. }\end{array}$ & $\begin{array}{c}\text { FRF based } \\
\text { SEREP }\end{array}$ \\
\hline Mode 1 & 20.2 & 21.46 & 24.1 & 27.0 \\
Mode 2 & 25.2 & 25.55 & 28.0 & 30.5 \\
Mode 3 & 51.5 & 49.99 & 50.5 & 52.1 \\
Mode 4 & 55.0 & 55.73 & 57.0 & 57.6 \\
Mode 5 & 72.5 & 68.37 & 71.1 & 71.4 \\
Mode 6 & 81.5 & 76.00 & 79.4 & 87.5 \\
Mode 7 & 103.0 & 100.59 & 100.2 & 101.0 \\
Mode 8 & 110.1 & 113.70 & 111.0 & 112.0 \\
Mode 9 & 131.9 & 131.48 & 130.1 & 137.2 \\
\hline
\end{tabular}

frequencies and mode shapes (only translational components are required). The hybrid coupling procedure outlined by Eqs (6) and (13) is applied, by truncating the number of modes representing the dynamic behavior of the larger plate to $m=20$ and by using $N_{G}=8$ interface modes. (Also, the first natural frequency for fixed test DoFs is $744 \mathrm{~Hz}$, ensuring a fair accuracy of the expansion technique). To try to reproduce the effect of measurement errors, the theoretical mode shapes are polluted with noise: a uniform random distribution is used for each modal vector component, with a maximum deviation ranging from $10 \%$ to $30 \%$ of the theoretical value. In Table 4 , the natural frequencies predicted by the structural modification procedure are compared with those provided by the FE model of the modified structure.

The results are very good, except for the 5th and the 10th mode, which are in error even when noise free data are considered. Being the natural frequencies lower than expected, the error should be ascribed to the expansion procedure (modal truncation, similarly to an additional constraint, would have produced higher natural frequencies). However, the technique is quite robust to noise introduced into eigenvectors.

The procedure is then applied starting from the experimental FRFs measured on the base structure. The measured FRFs are used to identify the modal parameters of the original structure. The hybrid coupling procedure is applied again, by using $m=14$ modes for the base plate ( 3 rigid body modes +11 identified flexible modes) and by using $N_{G}=8$ interface modes. In Table 5, the predicted natural frequencies are compared with those identified from experimental tests on the modified structure. Furthermore, Table 5 shows the results obtained, using the same experimental data and the same modification, by the FRF based condensation procedure and by the FRF based SEREP expansion procedure.

It can be noticed that the modal based static expansion technique provides much better results than the FRF based dynamic reduction and SEREP, especially for the 1st and 2nd mode. An exception is represented by the 5th and 6th mode: while expansion is probably responsible for the 5th mode, nothing can be said about the 6th mode.

The FRFs of the modified structure can be synthesized in the required frequency range from the predicted modal parameters, by using theoretically derived upper and lower residuals. 


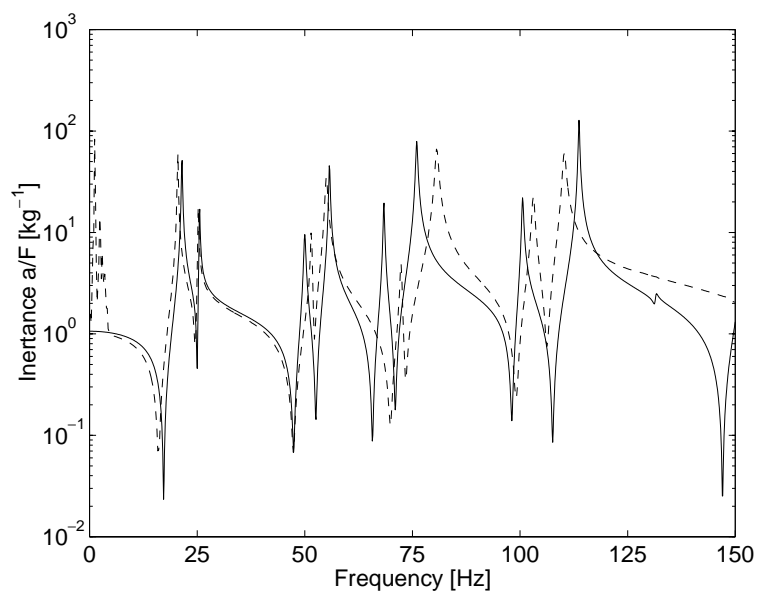

Fig. 8. FRF of the coupled structure: measured (-) vs. predicted (- - -) using static expansion and identified mode shapes.

In Fig. 8, an FRF measured on the modified structure is compared with the corresponding synthesized FRF. The agreement is satisfactory, at least for low frequencies.

\section{Conclusion}

When performing structure coupling, the knowledge of rotational DoFs is of paramount importance: in general, if they are neglected, meaningless results are obtained. The problem arises if any of the coupled substructures is known from experimental tests (FRFs or output-only tests), where it is quite difficult to measure the rotational DoFs.

In this paper some possibilities to deal with unmeasured rotational DoFs are analyzed. One solution is to estimate the rotational DoFs of the experimental substructure by expansion techniques from the measured translational DoFs. Among the many possibilities, the SEREP is a classical procedure that works independently of the connection considered, but has the drawback of requiring a theoretical model of the entire substructure. As an alternative to SEREP, the static expansion is well suited for line and surface connections and, thus, it can be used successfully for distributed structural modification in the low frequency range.

A second approach, that can be applied to point connections, is developed to avoid the use of rotational DoFS. An equivalent set of points, involving only forces and displacements, is used to model each point connection, involving also moments and rotations. Such model is capable of describing, through measurement of translational DoFs only, an average rotation of the joint.

The above procedures are tested on suitable benchmarks - one formed by a plate and a frame, the second one by a reinforcing plate coupled to a base plate - to deal in turn with point and surface connections. For the plate-frame benchmark the SEREP yields good results, provided that the number of test points is sufficiently low to avoid ill-conditioning. The equivalent multi-point modelling of the joint permits to obtain quite reliable results, at least in the low frequency range, indicating that this could be a promising alternative in practical situations. This is an appealing result because it indicates that it is possible in principle to use the procedure requiring only translational DoFs, thus avoiding the application of expansion techniques while obtaining a significant computational advantage.

For the reinforced plate benchmark, the application of the static expansion provides better results at low frequencies than the SEREP expansion and the dynamic reduction, confirming that a technique appropriately developed for a particular connection is usually more efficient than a general purpose technique.

\section{Acknowledgements}

This work is performed under MIUR (Italian Ministry of Education, University and Research) grant COFIN01. 


\section{References}

[1] D.J. Ewins and M. Sainsbury, Mobility measurement for the vibration analysis of connected structures, Shock and Vibration Bulletin 42 (1972), 105-122.

[2] A. Sestieri, P. Salvini and W. D'Ambrogio, Reducing scatter from derived rotational data to determine the frequency response function of connected structures, Mechanical Systems and Signal Processing 5 (1991), 25-44.

[3] F. Piergentili, P. Avitabile and J. O'Callahan, Dynamic expansion of frequency response functions, 17th International Modal Analysis Conference, Orlando, USA, 1999, pp. 769-778.

[4] W.C. Hurty, Dynamic Analysis of Structural Systems Using Component Modes, AIAA Journal 3 (1965), 675-685.

[5] R.R. Craig and M.C.C. Bampton, Coupling of Substructures for Dynamic Analysis, AIAA Journal 6 (1968), $1313-1319$.

[6] A.G. Kistler, Translational-Angular Piezo-Beam System, Datasheet no. K8.8832e, Ed. 4.01, 2001.

[7] L. Meirovitch, Principles and Techniques of Vibrations, Prentice Hall, 1997.

[8] A. Stanbridge and D.J. Ewins, Measurement of translational and angular vibration using a scanning laser Doppler vibrometer, Shock and Vibration 3 (1996), 141-152.

[9] M.J. Ratcliffe and N.A.J. Lieven, Measuring rotational DOFs using a Laser Doppler Vibrometer, Journal of Vibration and Acoustics 122 (2000), 12-20.

[10] M. Bello, A. Sestieri, W. D’Ambrogio and F. La Gala, Development of a rotation transducer based on bimorph PZT's, Mechanical Systems and Signal Processing 17 (2002), 1069-1081.

[11] K. Bendel, E. Brechlin and A. Storz, Electromagnetic vibration excitation of moments and forces with minimal mass loading, 25th International Seminar on Modal Analysis, Leuven, Belgium, 2000, pp. 967-972.

[12] Y. Champoux, O. Beslin, B. Gautier, D. Macheto and B. Paillard, Moment mobility measurement using an impact force couple, 25th International Seminar on Modal Analysis, Leuven, Belgium, 2000, pp. 989-996.

[13] M.W. Trethewey and H.J. Sommer III, Measurement of Rotational DoF Frequency Response Functions with Pure Moment Excitation, International Conference on Structural Dynamic Modelling, Funchal, Portugal, 2002, pp. 81-88.

[14] B. Jetmundsen, R.L. Bielawa and W.G. Flannelly, Generalized Frequency Domain Substructure Synthesis, Journal of the American Helicopter Society 33 (1988), 5-64.

[15] J.H. Gordis, R.L. Bielawa and W.G. Flannelly, A General Theory for Frequency Domain Structural Synthesis, Journal of Sound and Vibration 150 (1991), 139-158.

[16] S. Hou, Review of Modal Synthesis Techniques and a New Approach, Shock and Vibration Bulletin 40 (1969), $25-39$.

[17] S. Rubin, Improved Component-Mode Representation for Structural Dynamic Analysis, AIAA Journal 13 (1975), $995-1006$.

[18] R.R. Craig and C.J. Chang, On the Use of Attachment Modes in Substructure Coupling for Dynamic Analysis, 8th AIAA/ASME SSDM Conference Journal, 1977, pp. 89-99.

[19] W. D'Ambrogio, Some remarks about structural modifications involving additional degrees of freedom, Mechanical Systems and Signal Processing 4 (1990), 95-99.

[20] W. D'Ambrogio and A. Sestieri, Coupling theoretical data and translational FRFs to perform distributed structural modification, Mechanical Systems and Signal Processing 15 (2001), 157-172.

[21] J.C. O'Callahan, P. Avitabile and R. Riemer, System Equivalent Reduction Expansion Process (SEREP), 7th International Modal Analysis Conference, Las Vegas, USA, 1989, pp. 29-37.

[22] E. Balmès and L. Billet, Using Expansion and Interface Reduction to Enhance Structural Modification Methods, 19th International Modal Analysis Conference, Orlando, USA, 2001, pp. 615-621.

[23] E. D'Amato, F. Durante and W. D'Ambrogio, Assessment of interface modelling procedures for distributed structural modification, International Conference on Structural Dynamic Modelling, Funchal, Portugal, 2002, pp. 397-406. 

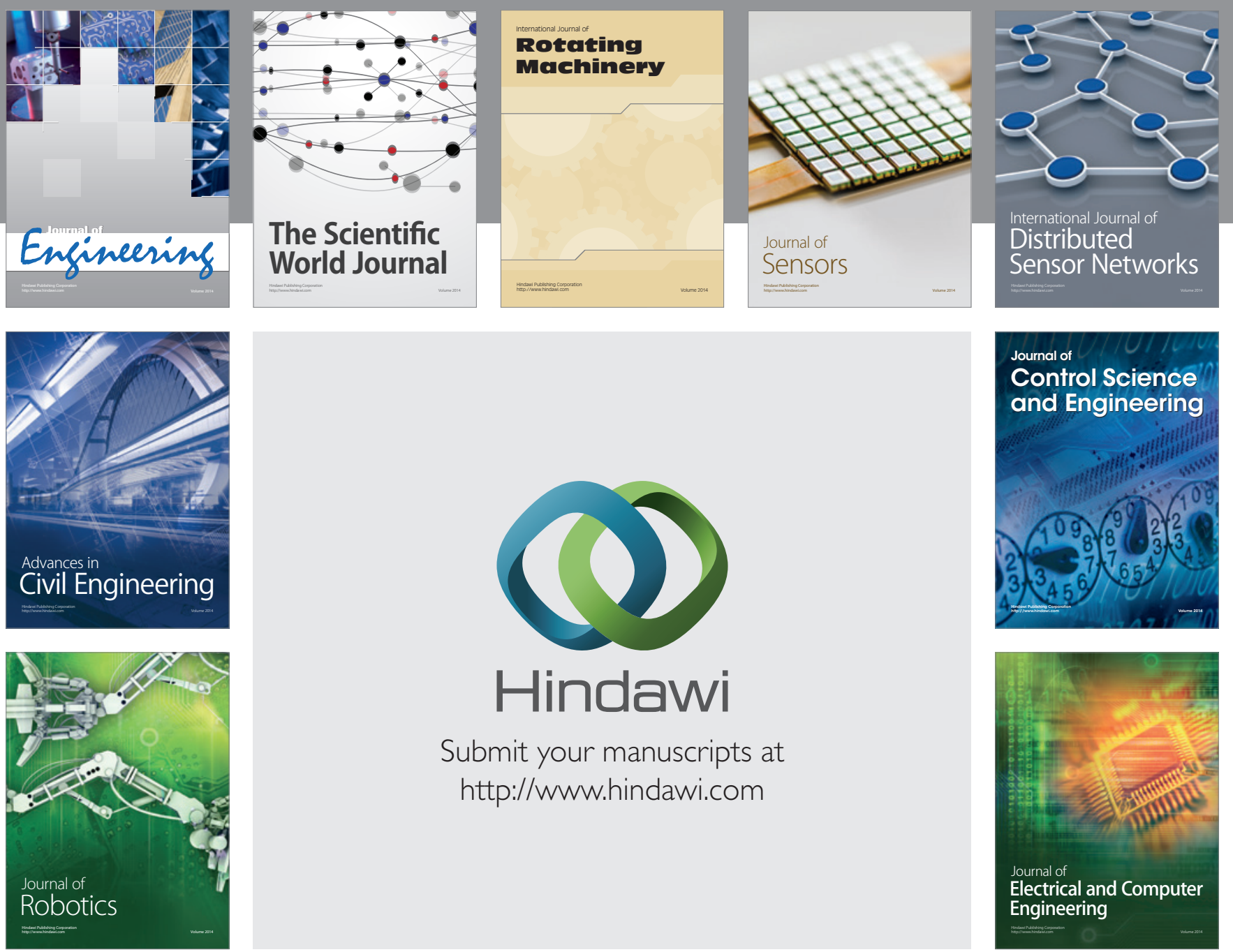

Submit your manuscripts at

http://www.hindawi.com
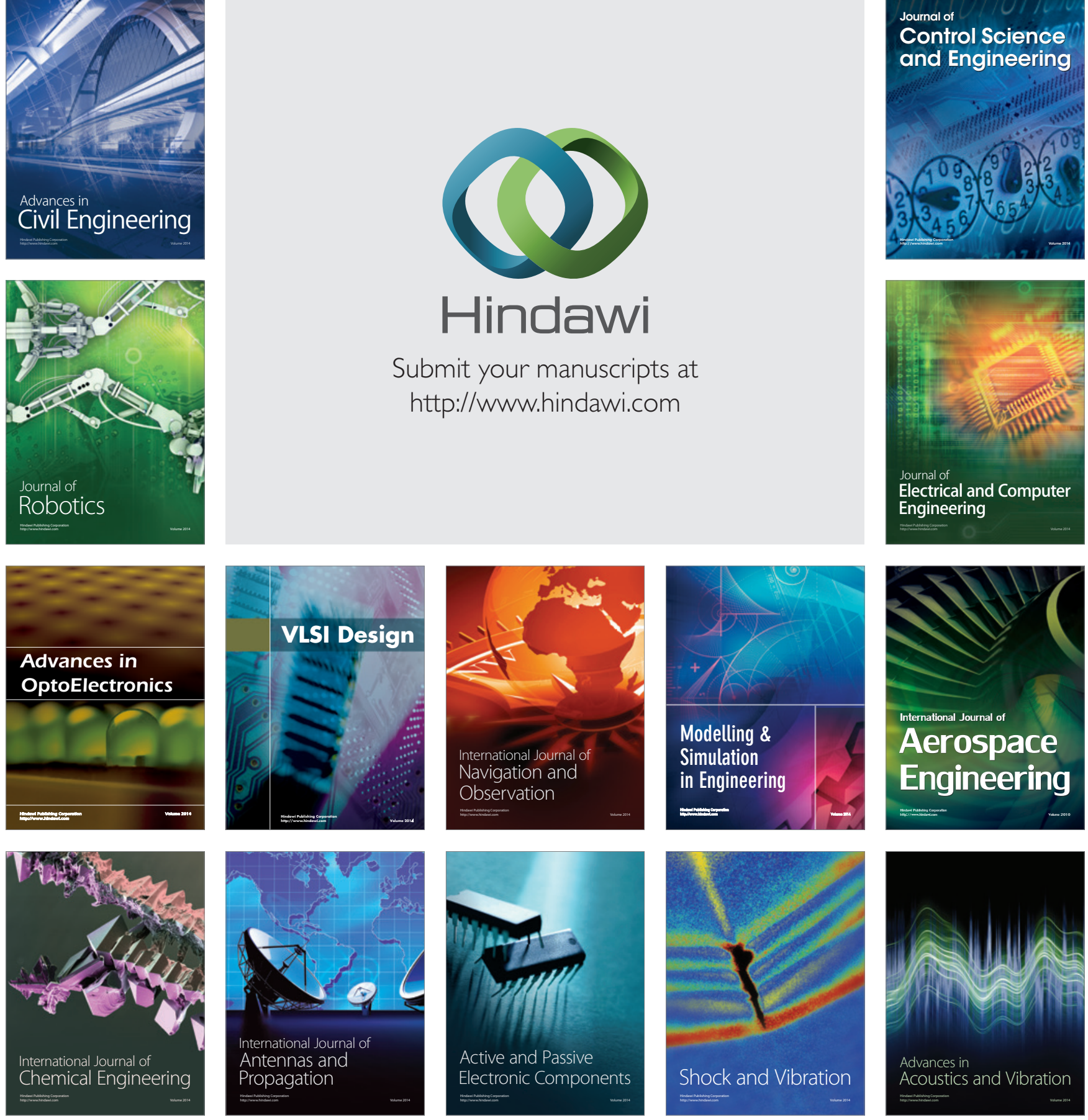